displayed high level of concordance (99.4-100\%). The prevalence of MG was $2.5 \%$ among females and $9.6 \%$ among males. The highest sensitivity $(71.4 \%-100 \%$ in different specimens) was exhibited by the AmpliSens rtPCR. All tests had a 100\% clinical specificity. The prevalence of TV was $1.2 \%$ among the females, and all additional VD patients tested positive. The sensitivity and the specificity of both Russian TV tests validated was 100\%.

Conclusion It seems clear that the biomedical industry in Eastern Europe has the potential for producing reliable reagents and tests kits at affordable prices for genetic diagnosis of STIs. This would open new perspectives for the whole region and could also be costeffective for some other regions experiencing financial constraints. However, more comprehensive evaluations of regionally manufactured tests should be conducted according to internationally accepted guidelines.

\section{LBP-1.05 CHALLENGES AND BARRIERS FOR CONDUCTING STI/HIV PREVENTION PROJECTS TARGETING FEMALE SEX WORKERS WITHIN NATIONAL PROGRAMS IN BENIN AND NIGER}

doi:10.1136/sextrans-2011-050119.11

${ }^{1} \mathrm{G}$ Batona, ${ }^{2} \mathrm{~A}$ Michel, ${ }^{3} \mathrm{~K}$ René, ${ }^{4} \mathrm{G}$ Marie, ${ }^{3} \mathrm{~A}$ Évelyne, ${ }^{3} \mathrm{~A}$ Clément, ${ }^{5} \mathrm{I}$ M Kamaye, ${ }^{5} \mathrm{D}$ Hassane. ${ }^{1}$ Université Laval, Unité de recherhe en santé des populations (URESP), Québec, Canada; ${ }^{2} U R E S P-C H A$, Université Laval, Québec, Canada; ${ }^{3}$ Programme National de lutte contre les IST/Sida du Bénin, Cotonou, Benin; ${ }^{4}$ Université Laval, Québec, Canada; ${ }^{5}$ Programme National de lutte contre les IST/Sida du Niger, Niamey, Niger

Context In West Africa, HIV/STIs prevention activities targeting female sex workers (FSW) and their partners were developed and supported financially through a bilateral cooperation project. After the end of the project in Benin (2006) and Niger (2007), the national HIV/STI programs took over these interventions.

Objectives (i) to identify the challenges and barriers for FSW program interventions within national programs; (ii) to assess the capacity of FSW dedicated services to treat and prevent STI efficiently.

Methods A triangulation of methods and sources of data collection were used within an evaluative approach centered on the use of the results, such as individual and collective interviews, review of data records from health centers and field observations. Quantitative data from clinical attendance were crossed with qualitative data. A conceptual framework was developed to explain the exploratory and analytical elements. The main findings were validated with the stakeholders.

Results There are several constraints and major challenges facing STI/HIV prevention under the responsibility of national programs, namely: (i) the deficit of synergies between two major components (communication for behavioural change and medical follow-ups), (ii) the lack of coordination and actions in the field, (iii) the abandonment of structural activities, (iv) low resource allocation for activities targeting FSW. Since the integration of the activities into the national programs, the capacity to provide prevention services to the FSW population, both in terms of coverage and of the package and the quality of services provided, has significantly declined, even if the strengthening of staff capacity in this domain remains an encouraging achievement.

Conclusions The national programs of Benin and Niger do not yet cover sufficiently the most exposed groups (FSW and their partners) who are at the centre of the HIV epidemic. Thus, the study proposes reflection and action to improve coverage of this clientele in order better control of STIs and HIV.

\section{LBP-1.06 INFECTIOUS SYPHILIS IN NEW BRUNSWICK: USING DATA FOR ACTION IN A SMALL CANADIAN PROVINCE}

doi:10.1136/sextrans-2011-050119.12

${ }^{1} \mathrm{G}$ Frosst, ${ }^{2} \mathrm{~F}$ W Tremblay, ${ }^{2} \mathrm{D}$ Allard. ${ }^{1}$ Public Health Agency of Canada, Fredericton, Canada; ${ }^{2}$ New Brunswick Department of Health, Canada

Background New Brunswick (NB) has experienced a resurgence of infectious syphilis with a 10-fold increase in the incidence rate between 2007 and 2010. In response to this increase in cases, an outbreak control team was convened and enhanced surveillance of syphilis was implemented to better understand the epidemiology of infectious syphilis in NB and to inform public health action.

Methods A standardised enhanced surveillance investigation form was developed to collect detailed information about syphilis cases, their sexual contacts, and risk factors. Since December 2010, all new cases of infectious syphilis reported in NB are followed up by regional Public Health staff using the standardised form, where completed forms are faxed to the provincial office for entry into a central database. Results are disseminated regularly to stakeholders through descriptive epidemiologic reports. Social network analysis (SNA) of cases, contacts, and meeting places for sexual partners (ie, venues) is also occurring to identify routes of transmission and points of intervention.

Results Fifty-six cases of infectious syphilis have been reported in NB since November 2009, corresponding to annual incidence rates of 5.0 per 100000 in 2010 and a projected 6.1 per 100000 in 2011. The majority of cases are located in the small urban centers of Moncton and Fredericton with sporadic cases located in Saint John and rural NB. Ninety-three per cent of cases are male with the highest incidence rate among males aged $20-24$ years (58.8 per 100000 population from November 2009 to April 2011). Five male cases are coinfected with HIV. Eighty-eight per cent of male cases are men who have sex with men, most of whom reported having multiple casual or anonymous sex partners in the months preceding diagnosis. Venues for meeting sex partners include websites, bars, and bathhouses. The epidemiologic reports and SNA informed the first phase of a province-wide social media campaign launched in February 2011. The campaign targets men aged $18-55$ years and includes distribution of posters, condom matchbooks, and online advertisements at venues identified by cases.

Conclusions NB is currently experiencing an outbreak of infectious syphilis. Enhanced surveillance activities, regular epidemiologic reporting, and SNA have informed the development of public health interventions targeting adult males, primarily men who have sex with men in Moncton and Fredericton.

\section{LBP-1.07 ECOLOGY OF HUMAN PAPILLOMAVIRUS (HPV) INFECTIONS IN THE MALE, STUDIED USING EXPRESSED PROSTATE SECRETIONS (EPS)}

doi:10.1136/sextrans-2011-050119.13

${ }^{1} \mathrm{~V}$ Smelov, ${ }^{2} \mathrm{C}$ Eklund, ${ }^{2} \mathrm{~J}$ Dillner. ${ }^{1}$ Medical Academy of Postgraduate Studies, St. Petersburg, Russian Federation; ${ }^{2}$ Karolinska Institutet, Stockholm, Sweden

Background In a new era of Human papillomavirus (HPV) vaccination, adequate diagnostics of the viral infection in men becomes more important: the monitoring of the vaccination effect requires evaluating whether vaccine HPV types disappear from and how the prevalence of non-vaccine types is affected in either high-risk groups or general population. Detection methods and anatomical sites for optimal HPV sampling are of high interest among healthcare specialists. Expressed prostate secretion (EPS) obtained during digital rectal examination - a daily routine urological diagnostic procedure-and following massage of the prostate, represents an 
alternative sampling material for the study of HPV ecology in the male. We present the results from the first study on HPV detection in EPS, as detected using a reference method used at the WHO HPV LabNet global reference laboratory, a multiplex high-throughput genotyping using the Luminex system.

Methods The EPS samples were from 186 heterosexual men (mean age 32 (range 19-60) years (median sexual life start at 17.6 (9-25) years; 33 (1-500) median life-time sex partners; no concurrent STDs at the time of the study) were collected in a urology outpatient unit in St. Petersburg and tested for the presence of HPV DNA in Stockholm by the Luminex assay.

Results The results are presented in the Abstract LBP-1.07 table 1. HPV prevalence in the study population was $25.3 \%$ for all HPV types with $11.8 \%$ for oncogenic types only. High-risk (HR-) HPV 16 and 66 were the most common types 3.7 and $2.7 \%$ of men, respectively. Interestingly, untypable HR-HPV types were found in $3.2 \%$ of EPS samples. Multiple oncogenic types were found in $22.7 \%$ of all HR-HPV + EPS samples.

Conclusions EPS can be used for HPV studies in men: additional investigation of EPS may result in better understanding the transmission of HPV infection and to develop strategies for HPV prevention. For the first time, the full range of HPV types in the EPS of men with no STDs was explored.

Abstract LBP-1.07 Table 1 HPV infection prevalence in the prostate (EPS) samples of 186 men

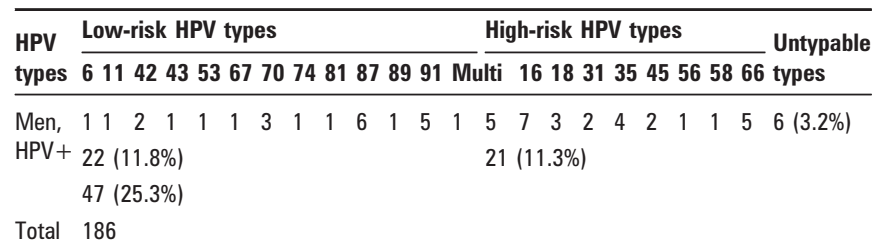

\section{LBP-1.08 PRELIMINARY REPORT ON EXPERIENCE WITH POINT OF CARE SYPHILIS AND HIV TESTING IN HARD-TO-REACH POPULATIONS IN OUTREACH SETTINGS IN EDMONTON, CANADA}

doi:10.1136/sextrans-2011-050119.14

\author{
${ }^{1} \mathrm{~J}$ Bergman, ${ }^{2} \mathrm{~S}$ Plitt, ${ }^{1} \mathrm{~J}$ Gratrix, ${ }^{1} \mathrm{P}$ Conroy, ${ }^{1} \mathrm{~J}$ Li, ${ }^{1} \mathrm{~K}$ Rocco, ${ }^{3} \mathrm{~J}$ Fenton, ${ }^{2} \mathrm{C}$ Archibald, \\ ${ }^{2} T$ Wong, ${ }^{1}$ A Singh. ${ }^{1}$ Alberta Health Services, Edmonton, Canada; ${ }^{2}$ Public Health \\ Agency of Canada, Ottawa, Canada; ${ }^{3}$ Provincial Laboratory for Public Health, \\ Edmonton, Canada
}

Background Edmonton is in the midst of a syphilis outbreak occurring largely in high risk and hard-to-reach populations. Point of care (POC) testing for syphilis and HIV offers the opportunity for immediate and rapid access to testing, and in the case of syphilis, immediate treatment. POC syphilis tests have not been previously evaluated in clinical settings in Canada.

Methods Since 14 February 2011, treponemal syphilis (Bioline Syphilis 3.0) and HIV (INSTI HIV-1/HIV-2 Antibody Test) POC testing, using whole blood from a finger prick specimen, has been offered to outreach clients in Edmonton. POC results were compared to standard testing from simultaneously collected serum specimens. Baseline demographics, sexual and drug use risk behaviour information were collected. Age and gender were collected on individuals who refused POC testing. A descriptive analysis was performed on the characteristics and outcomes of participants and those who refused.

Results As of 15 April 2011, 146 individuals had been offered POC testing; $85.6 \%(n=125)$ consented. Among participants, 59.2\% (74) were male [vs $66.7 \%$ (14) of non-participants, $\mathrm{p}=0.5)$ ] and the median age was 29 yrs (IOR 24-36 yrs) [vs non-participants median age $29 \mathrm{yrs}$ (IOR $25-45 \mathrm{yrs}$ ), $\mathrm{p}=0.2)$ ]. The majority of participants $(83.2 \%$; 104) were heterosexual, $59.2 \%$ (69) were Aboriginal, and $30.4 \%$ (38) reported injection drug use. Among females, $62.7 \%$ (32) reported sex trade involvement, while among males, $21.6 \%$ (16) reported sex with a sex trade worker. Of 121 syphilis treponemal POC tests, 5 (4.1\%) were positive, all in old treated cases of syphilis that were asymptomatic for infectious syphilis at the time of testing. Two syphilis POC $(1.7 \%)$ tests were falsely negative when compared to the standard screening test (Architect Syphilis TP Microparticles, Abbott Laboratories, Illinois, USA); both were in individuals previously treated for syphilis. Of 123 HIV POC tests, 2 (1.6\%) were reactive, both newly diagnosed cases as confirmed by GS HIV-1 Western Blot (BioRad Laboratories, California, USA); both were negative by syphilis POC tests.

Conclusion Preliminary results from Edmonton suggest that POC testing for syphilis and HIV is well accepted among high-risk populations in outreach settings in Edmonton. This ongoing study will assess the utility of these tests in mitigating the further spread of both syphilis and HIV through POC testing, and in the case of syphilis, POC treatment.

\section{LBP-1.09 SERVICES UTILISATION AND HEALTH RELATED VARIABLES OF PERSON LIVING WITH HIV/AIDS IN COMMUNITY IN TAIWAN}

doi:10.1136/sextrans-2011-050119.115

${ }^{1} \mathrm{P}$ Y Chiou, ${ }^{2} \mathrm{~L} \mathrm{C}$ Lin. ${ }^{1}$ Mackay Medicine, Nursing and Management College, Taipei City, Taiwan, China; ${ }^{2}$ Yang-Ming University, Taipei City, Taiwan, China

Background Non-profit organisation (NPO) is a major type to provide the services to response the needs for people living with HIV and AIDS (PLWHA) in the community in Taiwan. The purposes of the study were to describe the services and investigate the effect of the services utilisation of the services on health related variables for PLWHA.

Methods Stratified random sampling and cross-sectional survey methods were used. The self-administered questionnaires include the Demographic, Utilisation and Satisfaction of the Non-Profit Organisation Services Questionnaire, CD4 count and virus load, Customised Adherence Self-Report Questionnaire, Beck Depression Inventory-II (BDI-II), and Medical Outcomes Study Short-Form Health Survey (SF-36) were used to collect date. In Utilisation and Satisfaction of the Non-Profit Organisation Services Questionnaire, the nine services form NPOs were generalised. Data were analysed using forward stepwise multiple regressions.

Results There were 202 study subjects ( $n=178,88.1 \%$ male) and the mean age was 42.54 (SD 10.44) years. The mean virus load and CD4 count were 11031.07 (SD 67153.41) $\mathrm{m} / 1$ and 425.03 (SD 209.30) $\mathrm{mm}^{3}$. The total number of service types which had had accepted by participants was 4.65 (SD 1.67). The services with highest number of users were group or activities $(n=174,86.1 \%)$ and counselling ( $n=137,67.8 \%$ ). Of the 137 participants who had used the counselling service, 126 (92\%) will keep using counselling service. The result indicated that higher frequency of financial aid and counselling services were predictive to the lower depression level of BDI-II $(\mathrm{F}=17.51, \mathrm{R} 2=0.60, \mathrm{p}=0.001)$. Higher frequency of access for medical care service could predict the higher adherence rate to cART of Customised Adherence Self-Report Questionnaire ( $F=6.85, \mathrm{R} 2=0.41$, $p=0.013)$. Higher frequency of access for medical care service and higher adherence rate for cART were collaboratively the predictors to the higher $\mathrm{CD} 4$ count $(\mathrm{F}=17.24, \mathrm{R} 2=0.55, \mathrm{p}=0.009)$. The service of access for medical care, BDI-II, and CD4 were significant with quality of life for SF-36 $(\mathrm{F}=17.31, \mathrm{R} 2=0.66, \mathrm{p}<0.000)$. 